\title{
Cytological screening and Seroincidence of IgM with possible risk factors associated with naturally selected high risk group for Oncogenic Human Papilloma Virus Infection in Daura, Katsina State
}

\author{
${ }^{1 *}$ Hayatudeen M.R, ${ }^{1}$ Abdulkadir B. Mukhtar A. and Aminu M. ${ }^{2}$ \\ ${ }^{1}$ Department of Microbiology, \\ Faculty of Sciences, \\ Umaru Musa Yar Adua University, Katsina. \\ ${ }^{2}$ Department of Microbiology. \\ Faculty of Science, \\ Ahmadu Bello University, Zaria
}

Email: hayatudeen.muhammad@umyu.edu.ng

\begin{abstract}
Cervical cancer is the second most common cancer among women in the developing countries and the seventh commonest cancer in the developed countries. Human papillomavirus (HPV) is now known to be the main factor in the aetiology of cervical cancer with over $99.7 \%$ of cases being associated with previous high risk HPV infection. The aim of the research is to determine the risk factors and incidence of oncogenic human papilloma virus among women of reproductive age in Daura. This was a cross-sectional prospective study involving a total of 120 women. Questionnaires were administered to collect data such as socio-demographic and possible risk factors. Endocervical swab and serum samples were then collected from the participants. Samples were analyzed by Enzyme Linked immunosorbent Assay and Pap smear respectively. The findings of this research revealed and overall high seroincidence of $36.6 \%$ as well as significant difference between seroincidence rate of pregnant and non-pregnant women. The findings also revealed that 10-20 age groups had the higher seroincidence rate of $(50.0 \%)$ with no statistical significant difference between age groups. None of the sociodermographic factors involved in the research show significant association with HPV infections. The result by type of marriage shows that similar seroincidence rate was obtained in both monogamy and polygamy forms of marriage (36.7\%). The study also reveals that risk factors like sexual partners, HIV status and sexual debut are significantly associated with HPV infection $(P \leq 0.05)$. Sixty (60) participants show normal cytole under microscope with prevalence of $50.0 \%$ and those with abnormal cytole had prevalence of $(16.7 \%)$ while lowest prevalence of $(3.3 \%)$ was obtained from participants with high squamous intraepithelial lesions. These results demonstrate a high seroincidence of HPV infection among women in the study area. We recommend improved education regarding HPV and cervical cancer among the populace and increasing cervical cancer screening particularly to attendees of antenatal care to prevent HPV related morbidity and mortality.
\end{abstract}

Keywords: HPV; Cervical cancer; Endocervical swab; Seroincidence 


\section{INTRODUCTION}

Human Papilloma virus (HPV) accounts for most cases of cervical cancer with high risk HPV (hrHPV) serotypes largely responsible. Papillomaviruses are ubiquitous and are members of a family Papillomaviridae of DNA viruses that infect humans and animals (AMC, 2014). Human papillomaviruses (HPVs) are small non-enveloped viruses that contain a double-stranded, closed circular DNA genome. HPVs are a group of more than 200 related viruses. More than $40 \mathrm{HPV}$ types can be easily spread through direct sexual contact, from the skin and mucous membranes of infected people to the skin and mucous membranes of their partners. They can be spread by vaginal, anal, and oral sex (Satterwhite et al., 2013). Other HPV types are responsible for non-genital warts, which are not sexually transmitted. More than 120 subtypes of HPVs have been classified based on their oncological potential of transforming cells as Low-risk HPVs, which do not cause cancer but can cause skin warts (Condylomata acuminata) on or around the genitals and anus (Satterwhite et al., 2013).

Globally, cervical cancer was the third most common cancer ranking after breast and colorectal cancer and the fourth most common cause of cancer death ranking below breast, lung, and colorectal cancer (Louie et al., 2009). However, cervical cancer ranked second of all cancers for women in the sub-Saharan African countries with the highest incidences and mortality rate in eastern and western Africa according to the International Agency for Research on Cancer 2008 (Jemal et al., 2011). Still, the total number of cervical cancer cases registered in African countries is the lowest in the world, and this may be attributed to differences in sexual practice, lifestyle, and genetic predisposition. Despite the lower number of registered cases, what is alarming is that most women with cervical cancer in African countries do not seek treatment until later stages of the disease.The cervical cancer burden in developing countries creates a genuine threat to the world because infectious diseases do not recognize borders. Unfortunately, developing countries have neitherscreening programs nor facilities to implement them, and some of these countries continue to rely on primitive detection techniques.

African countries and their estimated current population (in millions) with Nigeria has the highest population of over 170, 000, 000 but unfortunately, fewer than 50 articles examining HPV and cervical cancer have been published (Iliyasu et al., 2010).However, because cervical cancer is not among the most priotize diseases in the most of the African countries, the costeffectiveness of implementing HPV vaccination programs is debatable. Further evaluation will be required, especially given the current changes in socioeconomic status, lifestyles, and reproductive patterns of individuals in the Africa (Louie et al., 2009).

In Katsina State there is no adequate information on risk factors and prevalence of HPV infection especially among women of reproductive age within the community. There is also great shortage of trained healthcare personnel in the area and this is due to lack of career advancement, poor institutional support in terms of infrastructure, equipment, staff, access to training, and modern technologies such as medical equipment, access to electronic libraries, internet, among others.

\section{MATERIALS AND METHODS}

\section{Study Area}

The study was carriedout in Daura Local Government Area of Katsina State. It is the spiritual home of the Hausa people. The emirate is referred to as one of the "seven true Hausa states" (Hausa Bakwai). Daura has many hospitals and medical facilities, the 
Daura healthcare system is generally divided into public and private sectors which provide medical services at the primary and secondary levels. Although the private hospitals are usually more expensive, it does not necessarily translate to better healthcare delivery (Dauda et al., 2011).

\section{Sample Size}

A sample of 120 women aged 18 years and above among patients presented for Pap smear screening at General Hospital Daura and Comprehensive Hospital Daura was determined using the equation described by (Nainget al., 2006) based on a previously established prevalence rate at $97.2 \%$ attending STI clinics in Kano Nigeria (Auwal et al., 2012).

\section{Inclusion Criteria}

Women of reproductive age were included in the study (between the ages of 15yrs and above), only women who gave their consent were included in the research.

\section{Ethical Clearance and consent}

Ethical approval for the study was obtained from the ministry of health, Katsina State before commencement of the study. Consent was obtained from patients prior to recruitment in the study.Prior to sample collection a structured questionnaire was used to obtain information on sociodermographic, risk factors and clinical information on each enrollee.

\section{Blood and cervical swab sample collection}

A total of $120 \mathrm{blood}$ and cervical swab samples were aseptically collected concurrently using syringes and cytobrush with the assistance of professional health workers. The whole blood was allowed to clot for at least 30mins and then centrifuged at $1000 \mathrm{rmp}$ for $10 \mathrm{mins}$; the serum was then carefully removed with transfer pipette and transferred aseptically into a sterile labeled serum storage screw lapped container. It was then stored in a freezer for further analysis.

Cervical swab samples were collected as described by Manga et al., 2016. Briefly, the collection of the sample was done bya consultant pathologist in the presence of a female nurse who also served as a chaperone. In collecting the sample, the women were placed in lithotomy position, the cervix was exposed using the bivalve speculum and then inspected. The specimen for the Pap smear was collected using the cytobrush, cut short at the brush end and inserted into already labeled vial preservative containers, which contained a physiological saline. All collected samples with the cytobrush were immediately packed in an ice pack in a container and were sent to the Laboratory for Pap smear analysis. Finally, the collected cells were examined under a microscope to observe abnormalities in the cells.

\section{Serological Assay for IgM antibody}

The serum sample were analyzed using Human Papillomavirus Antibody IgM (HPV-IgM) specific ELISA kit (96T) manufactured by Melsin Medical Co., Limited, Kuancheng District, Jilin Province, China. The manufacturer's instruction were strictly followed. Briefly, polystyrene plate was treated with a solution of either antigen. An untreated protein-based solution was used to cover all unbound sites on the plates. Enzyme-conjugated antibody or antigen binds specifically to the target antigen or antibody. Substrate was added and the signal produced by enzyme-substrate reaction was measured. To obtain the cut off O.D value, the O.D of the calibrator was multiplied by the value of the factor printed on the label. The antibody index was calculated by dividing the O.D value of each sample by the obtained value of cut off. Antibody index less than 1.0 is considered negative while greater than or equal to 1.0 is considered positive. 


\section{RESULTS}

The data collected from the structured questionnaire and result from laboratory analysis was presented using descriptive statistics, and analyzed using the statistical tool Graph Pad Prism Statistical Software (GPPSS) version 69. The results were presented in both tables and charts. Chi-square test and Odds ratio were used at 0.05 significance level and at $95 \%$ confidence intervals to test for statistical association between HPV positivity and the risk factors for HPV infections.

Out of 120 participants recruited in the study, an overall seroincidence (36.6\%) of HPV IgM was recorded as shown in Figure 1. Analysis of data and result according to category of the participants as either pregnant or non-pregnant is shown in figure 2.The IgM seroincidence rates among pregnant women were $36.4 \%$ while IgM seroincidence rates among Nonpregnant women were $37.8 \%$. There was statistical significant difference between seroincidence rate of HPV infection among the pregnant and non-pregnant participants $\left(\chi^{2}=\right.$ 7.601, $\mathrm{df}=1, \mathrm{p}=0.044$ )as well those with no pregnancy are more confer to risk for HPV infection $(\mathrm{OR}=1.765,95 \%$ C.I $=1.765-3.412)$.

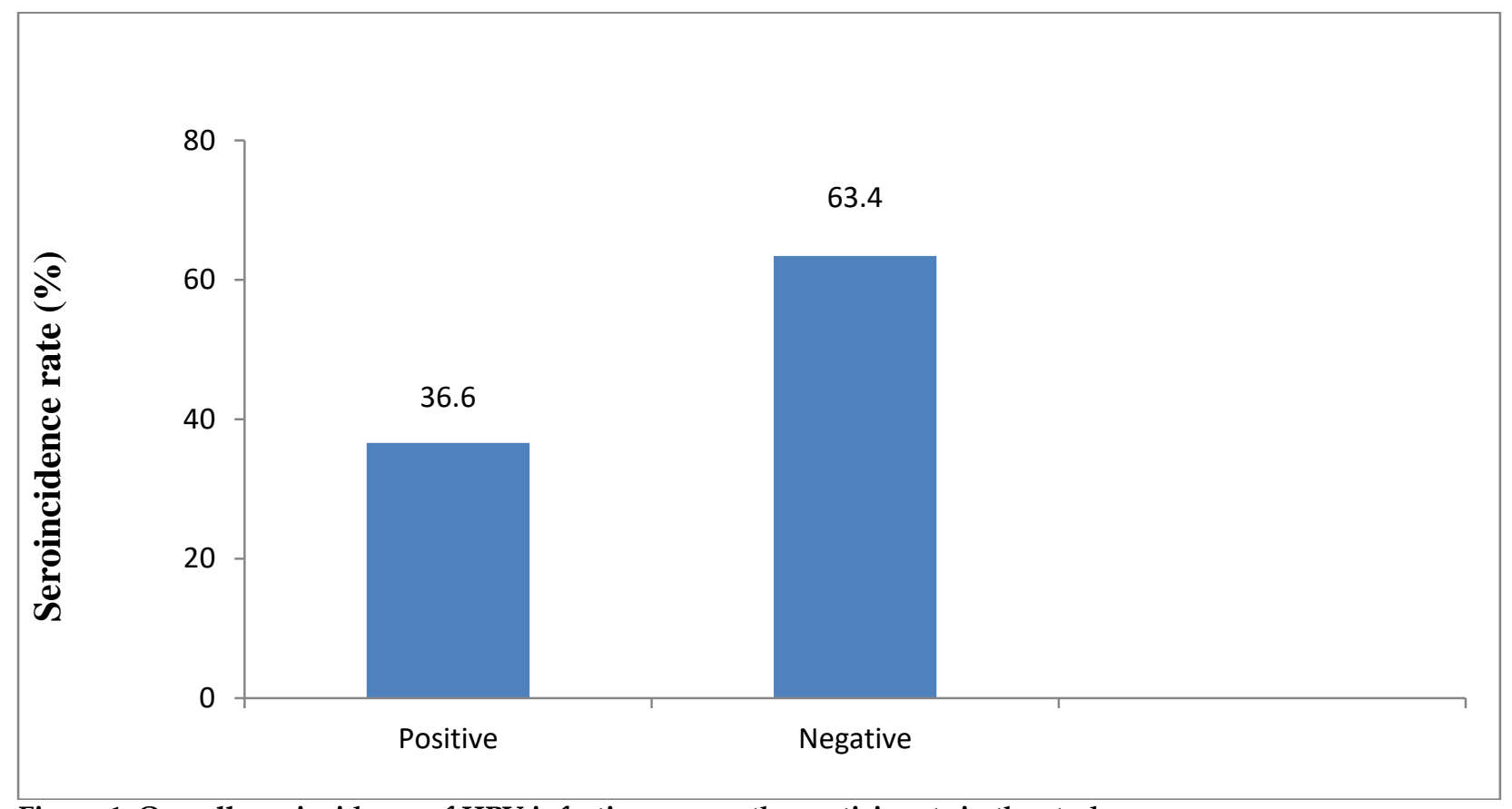

Figure 1: Overall seroincidence of HPV infection among the participants in the study area. 


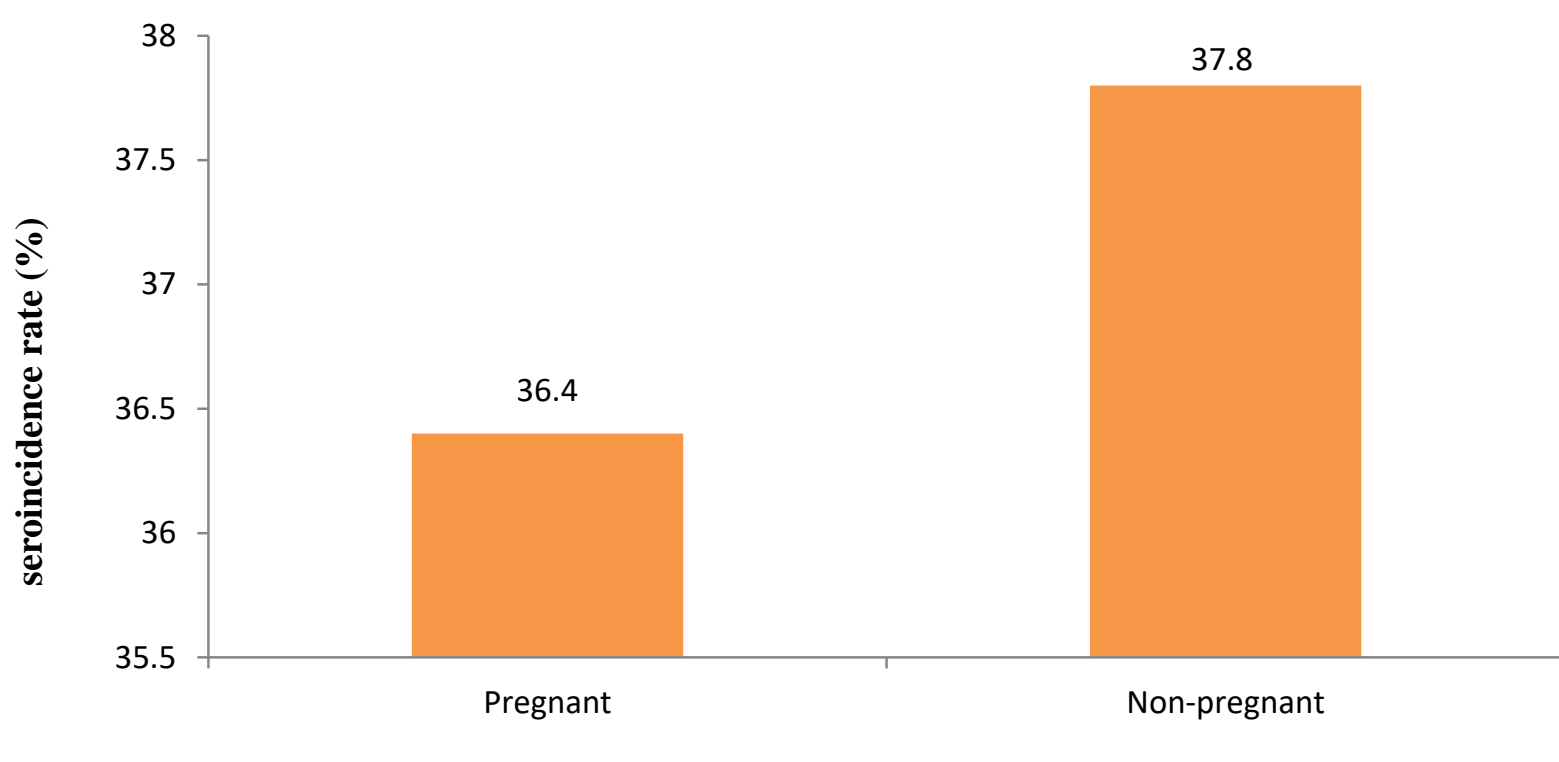

Figure2: Seroincidence of HPV Infection based on pregnancy status of the participants.

Analysis of data and result according to age group of the participant is shown in Table 2 were the higher seroincidence of $(50.0 \%)$ was recorded in 10-20 age group and lowest seroincidence was recorded among the 41-50 age group. There is no statistical significant association between age group and HPV infection. $\left(\chi^{2}=3.641, \mathrm{df}=3, \mathrm{P}=0.3030\right)$.

The result of HPV seroincidence in relation to socio dermographic factors is shown in Table3. Based on educational status, higher seroincidence(43.3\%)was recorded among those with secondary educational level while lower seroincidence $(5 \%)$ recorded among those participants with tertiary educational level. There is no statistical significant association between educational status and HPV infection $\left(X^{2}=2.127, \mathrm{df}=3, \mathrm{P}=0.5465\right)$. However, similar seroincidence was seen in both participants with monogamy and polygamy forms of marriage and no statistically significant difference $\left(\chi^{2}=0.0001, \mathrm{df}=1, \mathrm{P}=1.00021\right)$. Analysis of result by occupational status showed that higher seroincidence $(57.1 \%)$ recorded in skilled while lower in unskilled. There is nostatistical significant association between the occupation and HPV infection $\left(\chi^{2}=1.434, \mathrm{df}=3, \mathrm{P}=0.488\right)$.

Table 1: Seroincidence rate of HPV infection according to the age of the participants.

\begin{tabular}{llllll}
\hline Age group & Total participants & Positive $(\%)$ & Negative $(\%)$ & $\chi^{2}$-value & p-value \\
\hline $10-20$ & 24 & $12(50.0)$ & $12(50.0)$ & 3.641 & 0.3030 \\
$21-30$ & 78 & $30(38.5)$ & $48(61.5)$ & & \\
$31-40$ & 14 & $02(14.3)$ & $12(85.7)$ & & \\
$41-50$ & 04 & $00(0.0)$ & $04(100.0)$ & \\
Total & $\mathbf{1 2 0}$ & $\mathbf{4 4 ( 3 6 . 6 )}$ & $\mathbf{7 6 ( 6 3 . 3 )}$ & & \\
\hline
\end{tabular}


Table 2: Seroincidence rate of HPV infection based on the socio-demographic factors.

\begin{tabular}{|c|c|c|c|c|c|}
\hline Factor & Total number & Positive (\%) & Negative (\%) & $\chi^{2}$-value & p-value \\
\hline Education & & & & 2.127 & 0.547 \\
\hline None formal & 82 & $30(36.6)$ & $52(63.4)$ & & \\
\hline Primary & 10 & $04(40.0)$ & $06(60.0)$ & & \\
\hline Secondary & 22 & $10(45.5)$ & $12(54.5)$ & & \\
\hline Tertiary & 06 & $00(0.0)$ & $06(100.0)$ & & \\
\hline Others & 00 & $00(0.0)$ & $00(0.0)$ & & \\
\hline Type of marriage & & & & 0.00 & 1.00 \\
\hline Monogamy & 60 & $22(36.7)$ & 38 (63.3) & & \\
\hline Polygamy & 60 & $22(36.7)$ & $38(63.3)$ & & \\
\hline Occupation & & & & 1.434 & 0.488 \\
\hline House wife & 76 & $26(34.2)$ & $25(65.8)$ & & \\
\hline Skilled & 14 & $08(57.1)$ & $03(42.9)$ & & \\
\hline Unskilled & 00 & $00(0.0)$ & $00(0.0)$ & & \\
\hline Others & 30 & $10(33.3)$ & $10(66.7)$ & & \\
\hline
\end{tabular}

The data and result were analyzed according to risk factors as shown in Table 3. Participants with single partner recorded higher seroincidence rate $(41.6 \%)$ compared to those with multiple partners $(29.1 \%)$. There is statistical significant association between sexual partner and HPV infection $\left(\mathrm{X}^{2}=2.0071, \mathrm{df}=1, \mathrm{P}=0.033\right)$. Result according to parity shows those who are primiparous had higher seroincidence $(44.4 \%)$ while multiparous participants had lowest $(34.7 \%)$. There is no statistical significant association between parity and HPV infection $\left(\mathrm{X}^{2}=0.3287, \mathrm{df}=2, \mathrm{P}=0.849\right)$. The participants with $\mathrm{HIV}$ positivity had highest seroincidence rate $(52.6 \%)$ compared to those with HIV negativity $(29.3 \%)$. There is statistical significant association between HPV infection and HIV status $\left(\chi^{2}=2.129, \mathrm{df}=1, \mathrm{P}=0.045\right)$. The highest seroincidence rate of $(37.0 \%)$ was obtained among participants with younger age of sexual debuting compared to those with older age $(25.0 \%)$. There is a statistical significant association between age at sexual debut and HPV infection $\left(X^{2}=0.2512, \mathrm{df}=1, \mathrm{P}=0.016\right)$.

Table 3: Seroincidence rate of HPV IgM in relation to risk factorsin the study area

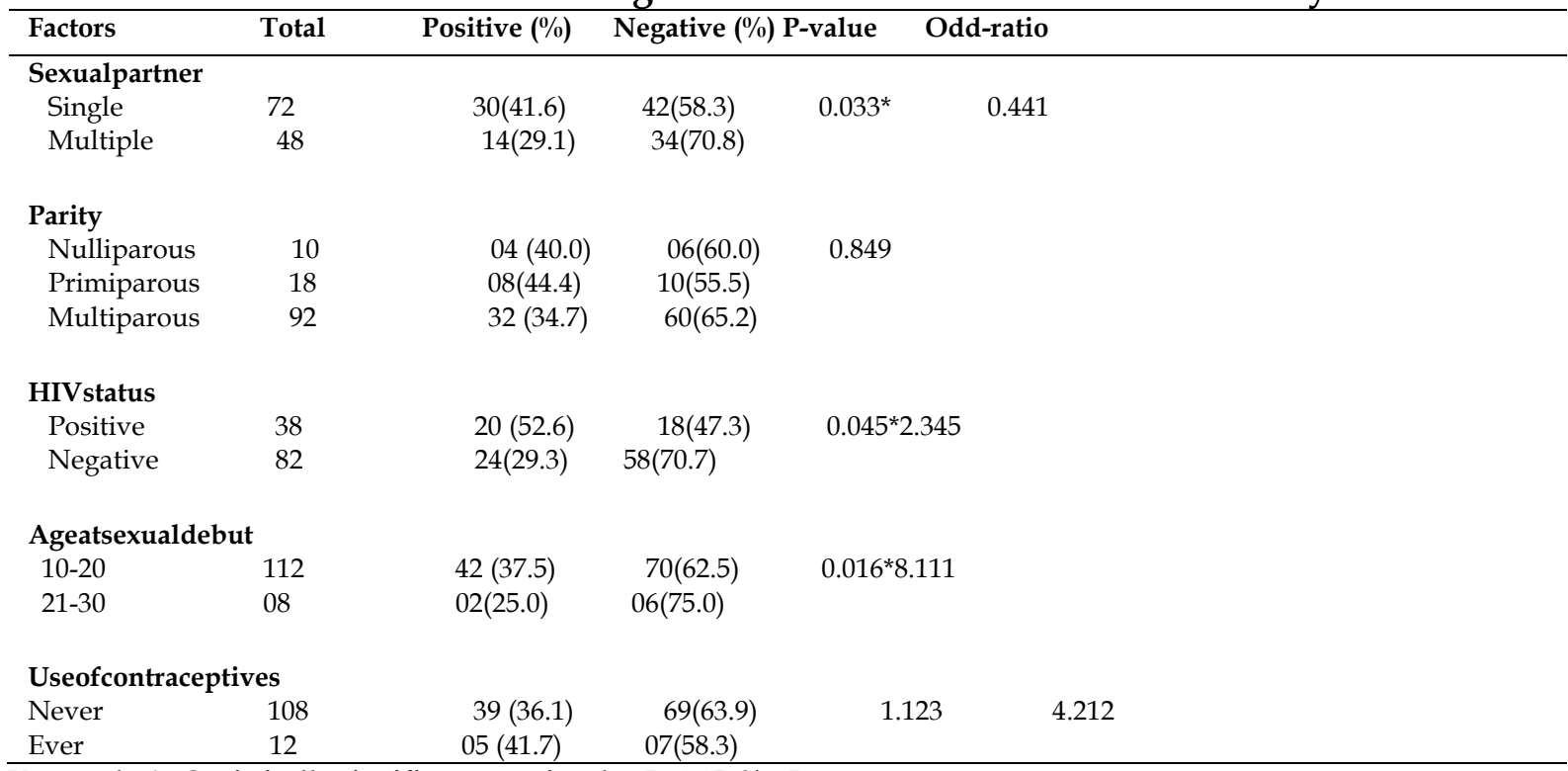

Keywords: * ${ }^{*}$ Statistically significant associated at $\mathbf{P} \leq 0.05, \%=$ Percentage 
Out of total of one hundred and twenty(120) women screened for cervical cancer $60(50.0 \%)$ had normal cytole, while $20(16.7 \%)$ had some form of abnormal cytology result, with ASCUS having highest percentage $(13.3 \%)$. The results obtained from Pap smear shows that there is significant difference between women with positive Pap smear and those with negativeresult.

Table 4:Occurrencesand Prevalences ofCervical Cytological findings of the participants.

\begin{tabular}{|c|c|c|}
\hline Cytological classes & & Prevalence (\%) \\
\hline Abnormal & 20 & 16.7 \\
\hline ASC-US & 16 & 13.3 \\
\hline LSIL & 12 & 10.0 \\
\hline HSIL & 04 & 3.3 \\
\hline SCC & 08 & 6.7 \\
\hline Normal & 60 & 50.0 \\
\hline Total & & 20100 \\
\hline
\end{tabular}

\section{Prevalence of abnormal cervical cytology (Pap smear) was 16.7\% at 95\% confidence} interval CI of (4.6-8.2).

ASC-US: Abnormal squamous cells of undetermined significant

LSIL: Low squamous intraepithelial lesion

HSIL: High squamous intraepithelial lesion

SCC: Squamous cells carcinoma

\section{DISCUSSION}

The study has provided important information on the seroincidence and risk factors associated with oncogenic HPV infection. The reported seroincidence of oncogenic HPV infection in this study is slightly higher than the seroincidence of HPV infection reported in Lagos, Nigeria (Adegbesan-Omilabuet al., 2014) but much higher than the seroincidence found in a study conducted in Ibadan, Nigeria (Clifford et al., 2005) and is also higher than the worldwide incidence of $10.4 \%$ (Sanjoseet al., 2003) and the rate obtained from other studies conducted in Asia (Pham et al., 2003) and North-America (Dunne et al., 2007).

These variations in the seroincidence rates may be contributed to the exhaustive nature of the HPV detection strategy used in the various studies especially those carried out in Asia and America and the number of oncogenic HPV types that were tested (AdegbesanOmilabuet al., 2014).

The finding of higher seroincidence of HPV infection among 21-30 years age group reported by this findingsis similar to finding conducted in Lagos (Adegbesan-Omilabuet al., 2014) and Ibadan (Thomas et al., 2004) where females of reproductive age were found to be highly susceptible to HPV infection.However, another study carried Conakry in Guinea showed an increased seroincidence rate of HPV among women who are less than 25 years of age (Keita et al., 2004). This may be due to fraction of the partners of these women may continue to have multiple sexual contacts either by practicing polygamy form of marriage or probably by indulging in extramarital affairs and thereby re-infecting themselves and their partners.

This study also revealed no significant association between the educational level and HPV infection. This agrees with the findings of (Nwekeet al, 2004) and may therefore;suggest that acquisition of HPV infection which is often thought to be related to sexual lifestyle may not necessarily be influenced by education as tendency to engage in unprotected sexual activities cut across all categories of participants irrespective of their educational status. 
Our study, also demonstrate any relationship between HPV infection and parity. This finding is mostly at variant with previous studies from within and outside the African continentthat showed that parity, use of oral combined oral contraceptive pills; HIV positivity and even smoking are all directly or indirectly related to increased sexual activities which may be related to HPV infection (Nwekeet al., 2004).

\section{CONCLUSION}

In conclusion, this study suggests a relatively high seroincidence rate (36.6\%) of high-risk HPV infection among women attending General Hospital Daura and Comprehensive Hospital Daura.The study demonstrated early age of sexual debut and increasing number of lifetime sexual partners as the most important risk factors associated with HPVinfections. The study revealed that there is high seroincidence in the participants with young age while the lowest among young age.The seroincidence rate of HPV infection based on risk factors revealed that individual that are single in relation to lifetime sexual partners has high HPV infection. Primigravity factor revealed that participants with young age had high proportion of the suspected HPV infection.

\section{REFERENCES}

Adegbesan-Omilabu M, Okunade K, Omilabu S. (2014). Oncogenic human papilloma virus infection among women attending the cytology clinic of a tertiary hospital in Lagos, South-West Nigeria. International Journal of Research in Medical Sciences ; 2:625.

American Cancer Society (2014). Cancer Facts \& Figures 2014Exit Disclaimer. Atlanta: American Cancer Society; 2014. Accessed February 25, 2014.

Auwal, I.K., Amin, M. Atanda, A.T., Tukur, J. \& Sarkinfada, F. (2013). Prevalence and risk factors of high risk human papillomavirus infections among women attending gynaecology clinics in Kano, northern Nigeria. Bayero Journal of Pure and Applied Sciences, 6(1): 67-71.

Clifford, G.M., Gallus, S., Herrero, R., Munoz, N., Sniders, P.J. and Vaccarella, S. (2005): Worldwide distribution of human papillomavirus types in cytologically normal women in the International Agency for Research on Cancer HPV prevalence surveys: a pooled analysis. Lancet. 366(9490): 991-998.

Dauda, U. Gulumbe, S.U., Yakubu, M. and Ibrahim L.K. (2011). Monitoring of Infectious Diseases in Katsina and Daura Zones of Katsina State: A Clustering Analysis. Nigerian Journal of Basic and Applied Science, 19(1); 31-42.

Iliyasu Z, Abubakar IS, Aliyu MH, and Galadanci HS (2010). Cervical cancer risk perception andpredictors of human papilloma virus vaccine acceptance among female universitystudents in northern Nigeria Journal of Obstetrics and Gynaecology, 30 (8):857-862.

Jemal,A.; Freddie Bray, Melissa M. Center, and Jacques F, (2011). Elizabeth Ward, David Forman.Global Cancer Statistics. Cancer Journal of Clinics, 61 (2): 69-90.

Louie K, Sanjose S and Mayaud P (2009). Epidemiology and prevention of human papillomavirus and cervical cancer in sub- Saharan Africa: a comprehensive review. TropicalMedicine and International Health, 14(10);1287-1302.

Manga M.M,, Adeola F, Yusuf M.A, Aliyu U.E, Danladi B.A, Hamidu U.P, Rasheed A.B, and Abimbola O.O (2016). Epidemiological patterns of cervical human papillomavirus infection among women presenting for cervical cancer screening in North-Eastern Nigeria, Infectious Agents and Cancer ;10:39.

Satterwhite C.L, Torrone E, and Meites E. (2013). Sexually transmitted infections among US women and men: Prevalence and incidence estimates. Sexually Transmitted Diseases, 40(3):187-193. 
Thomas J, Herrero R, Omigbodun A, Ojemakinde K, Ajayi !, Fawole A, Oladepo O, and Smith J. (2004). Prevalence of papillomavirus infection in women in Ibadan, Nigeria: apopulation-based study. British Journal of Cancer, 90: 638-645. 